\title{
A NEW ASYMPTOTIC METHOD FOR THE MODELING OF NEAR-FIELD ACCELEROGRAMS
}

\author{
By P. BERNARD AND R. MADARIAGA
}

\begin{abstract}
We study high-frequency radiation from a dislocation model of rupture propagation at the earthquake source. We demonstrate that in this case all the radiation emanates from the rupture front and, by a change of variables, that at any instant of time the high-frequency waves reaching an observer come from a line on the fault plane that we call isochrone. An asymptotic approximation to near-source velocity and acceleration is obtained that involves a simple integration along the isochrones for every time step. It is shown that wave front discontinuities (critical or stopping phases) are radiated every time an isochrone becomes tangent to a barrier. This leads to what we call the critical ray approximation which is given in a closed form. The previous results are compared with discrete wavenumber synthetics obtained by Bouchon (1982) for the Gilroy 6 recording of the Coyote Lake earthquake of 1980 . The fit between the asymptotic and full numerical method is extremely good. The critical ray approximation permits the identification of different phases in Bouchon's synthetics and the prediction of the behavior of the signal in the vicinity of their arrival time.
\end{abstract}

\section{INTRODUCTION}

Most methods to calculate synthetic acceleration and velocity in the vicinity of a seismic source rely on the numerical integration over the fault plane of complete near-field Green functions weighted by the slip functions. Several studies of radiation from dynamic source models have shown that it is possible to develop very efficient asymptotic methods for the simulation of accelerograms (Madariaga, 1977; Achenbach and Harris, 1978). Recently, we have found an analytical solution for the high-frequency radiation of a circular expanding crack, which stops abruptly and simultaneously around the circular rupture front (Bernard and Madariaga, 1983). The high-frequency approximation was then compared with solutions obtained by Archuleta and Hartzell (1981) and Campillo (1983) using standard integration of complete Green functions over the circular fault. The agreement between the two types of calculations was striking. The circular crack model, because of its simplicity, has been used as elementary source for modeling the near field of large events (Boatwright, 1982; Papageorgiou and Aki, 1983). But this model is clearly unphysical: a propagating circular rupture front would never stop simultaneously around its periphery, because there is little chance that it will encounter a preexisting circular barrier centered at the focus.

In this paper, we continue our investigation of asymptotic methods using only the far-field term of the Green function in the representation theorem. This approximation leads to a number of simplifications of the calculations and simple physical models of the generation of high-frequency waves. We assume that the seismic source is a dislocation with a propagating circular rupture front stopping abruptly on a preexisting barrier of arbitrary shape. We show that the barrier geometry controls the high frequency of the source, and give it a simple analytical form. 


\section{Geometrical Analysis of High-Frequency Radiation}

We are interested in high-frequency radiation from a dislocation or crack-like seismic source in which there is a strong concentration of slip velocity near the rupture front. By high-frequency radiation, we mean that the wavelengths in which we are interested are all shorter than the shortest distance from the observer to the source. In this case, the far-field term of the radiation from a point dislocation source is the appropriate Green function to calculate synthetic seismograms. Following Aki and Richards (1980), the far-field $P$ or $S$ wave are given by

$$
\boldsymbol{G}(P, t \mid 0)=\frac{1}{4 \pi \rho c^{3}} \boldsymbol{R}^{c} \frac{1}{D} \dot{M}_{0}(t-D / c)
$$

Here, $\rho$ is the density, $c$ stands for $\alpha$ or $\beta$ the compressional and shear wave velocities according to the particular wave under consideration, $D$ is the distance between the observer at $P$ and the source at $0, M_{0}(t)$ is the seismic moment, and $\boldsymbol{R}^{c}$ is the radiation pattern for $P$ or $S$ waves.

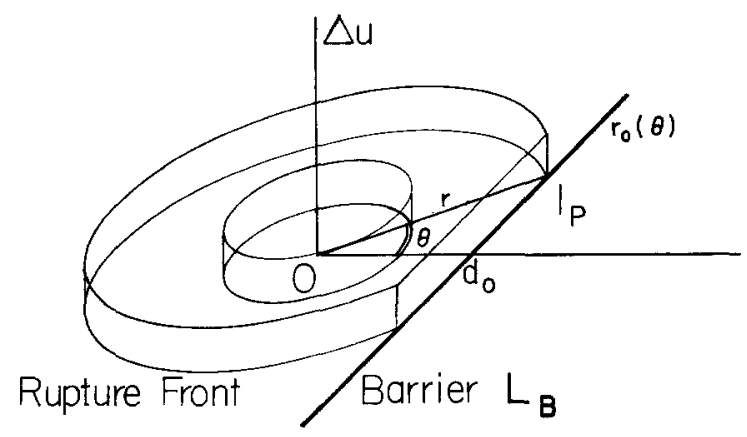

FIG. 1. The dislocation model with a straight barrier. The slip is constant inside the rupture front, which expands circularly with constant velocity in the fault plane, and stops when it reaches the barrier $L_{B}$.

In order to establish the basic results, we will use a very simple model for the source (Figure 1): The rupture initiates at the focus 0 and expands radially with constant velocity $v$ on the fault plane. The slip $\Delta u$ will be assumed to be uniform, constant, and parallel to the fault plane inside the rupture front limits. This simple model of the slip function is not physically acceptable but it may be converted into a crack-like dislocation by convolution as we will show later in the paper. Let the rupture grow until it reaches a straight-line barrier $L_{B}$ at distance $d_{0}$ from the origin 0 (see Figure 1). The slip and the slip velocity are expressed as

$$
\Delta u(r, \theta, t)=D_{0} H(t-r / v) H\left(r_{0}(\theta)-r\right)
$$

and

$$
\Delta \dot{u}(r, \theta, t)=D_{0} \delta(t-r / v) H\left(r_{0}(\theta)-r\right)
$$

respectively. Here $t$ is time, $r$ and $\theta$ are polar coordinates on the fault plane, $H$ is the Heaviside function, $\delta$ is the Dirac function, and the equation $r=r_{0}(\theta)$ is the 
analytical representation of the barrier line $L_{B}$. For a straight barrier line, we have

$$
r_{0}(\theta)=d_{0} / \cos \theta
$$

The slip velocity (3) is zero everywhere on the fault plane, except on the rupture front, where it becomes infinite. This is the model with the maximum possible concentration of slip velocity in the vicinity of the rupture front. In more realistic models, the concentration will be spread in a way determined by the conditions at the rupture front. For perfectly sharp cracks, $\Delta \dot{u}$ will have an inverse-square root singularity (Madariaga, 1977); for cracks with cohesion the singularity will be smoother (Achenbach and Harris, 1978). In Haskell's dislocation model the slip velocity singularity is spread over the rise time $\tau$, so that $\Delta \dot{u}$ will have a boxcar shape. Solutions for these kind of slip velocity singularities may be obtained by convolution of our solutions with appropriate source time functions.

In order to calculate the radiation from our source, we use the representation theorem for a flat seismic fault

$$
\boldsymbol{u}(P, t)=\int \boldsymbol{G}\left(P, t \mid \boldsymbol{r}_{0}\right) * \mu \Delta \dot{u}\left(\boldsymbol{r}_{0}, t\right) d S
$$

where $G$ is the Green function for a unit seismic moment with a step source time function, and $\boldsymbol{r}_{0}$ is the position on the fault. Using (1) we obtain

$$
\boldsymbol{u}^{c}(P, t)=\frac{\mu}{4 \pi \rho c^{3}} \int_{S} \boldsymbol{R}^{c} \frac{1}{D} \Delta \dot{u}(t-D / c) d S
$$

which is similar to equation (14.4) of Aki and Richards (1980). In (6) however, $\boldsymbol{R}^{c}$ and $D$ vary with position on the fault, i.e., we do not make the Fraunhoffer approximation. There is some confusion in the literature between far-field and high-frequency approximations as exemplified by equation (1), and the Fraunhoffer or far-from-the-source approximation which applies when the observer is at a distance far greater than the dimensions of the source. Our intention is to prove that within the limits of our theory, accelerograms calculated using (6) are very good approximations to those calculated with full wave theory including "nearfield" terms in the Green function (1).

Let us introduce the slip velocity (3) into (6) to obtain

$$
\boldsymbol{u}^{c}(P, t)=\frac{\mu D_{0}}{4 \pi \rho c^{3}} \int_{S} \boldsymbol{R}^{c} \frac{1}{D} \delta[t-\tau(P, r)] H\left(r_{0}(\theta)-r\right) d S
$$

where

$$
\tau(P, r)=r / v+D / c
$$

is the retarded time. Given a point on the fault $(r, \theta)$, the distance $D$ to the observer at $P$ is

$$
D(r, \theta)=\left(r^{2}+R^{2}-2 r d \cos \theta\right)^{1 / 2}
$$

where $r, \theta, R$ and $d$ are defined in Figure 2. 
Ignoring for the time being the barrier at $r=r_{0}(\theta)$, we may interpret (7) as the integral along a line on the fault defined parametrically by the equation

$$
t=\tau(P, r)=r / v+D(r, \theta) / c .
$$

For a given value of the time $t$ at the observer, equation (10) defines a closed curve on the fault plane that we call the isochrone. This curve defines the set of points $\left[r_{1}(t, \theta), \theta\right]$, solution of $(10)$ on the fault plane, from which radiation is arriving at $P$ at time $t$. Let us note that the points on the isochrone do not radiate simultaneously but only when the rupture front passes through them, i.e., at time $r_{1}(t, \theta) / v$. Following Figure 2, we can give a simple geometrical interpretation to

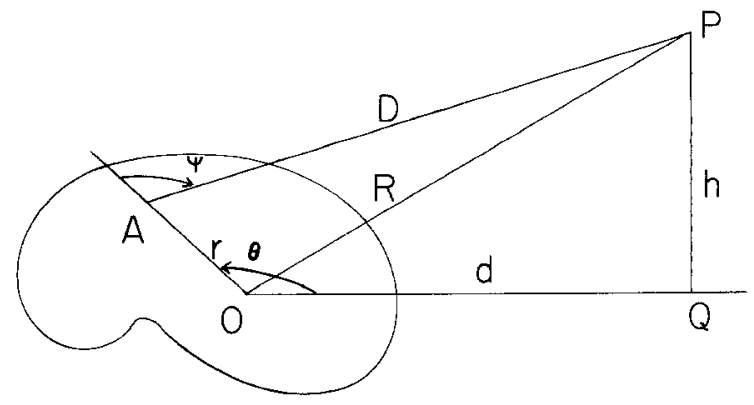

FIG. 2. Three-dimensional space geometry of the dislocation model. The observer is at $P$, whose projection on the fault plane is $Q$. Point 0 is the focus, $(r, \theta)$ are polar coordinates; $\phi$ and $D$ are the radiation angle and distance from point $A$ to the observer at $P$.

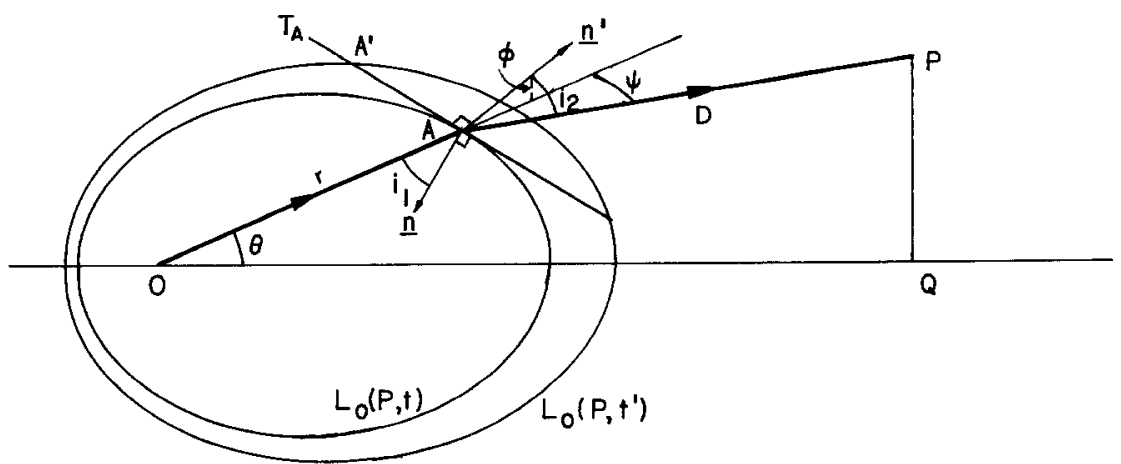

FiG. 3. Geometrical properties of the isochrones. Any point $A$ on the isochone $L_{0}(P, t)$ refracts the ray $O A$ in the direction of the observer at $P$ : angles $i_{1}$ and $i_{2}$ are related by Snell's law. $T_{A}$ is the tangent to the isochrone at the point $A, \boldsymbol{n}$ is the normal to $T_{A}$ on the fault plane, $\boldsymbol{n}^{\prime}$ is the normal $T_{A}$ contained on a plane through $T_{A}$ and $P . \phi$ is the angle of radiation entering into the directivity term.

(10) which will be useful in understanding radiation: $t$ is the travel time of a "rupture ray" that leaves the origin 0 in the direction $\theta$ with velocity $v$ and propagates for a distance $r$ along the fault. At the point $A$, of coordinates $\left(r_{1}, \theta\right)$, it leaves the source and propagates to the observer at $P$ as an elastic wave with velocity $c$. Then $r / v$ in (10) is the travel time of the rupture ray between 0 and $A$; and $D / c$ is the travel time along $A P$.

The equation for the isochrone in a homogeneous medium (7) may be easily solved numerically for $r=r_{1}(t, \theta)$. Let $L_{0}(P, t)$ be this isochrone which is a function both of the position of $P$ and the time of observation $t$. In a general medium, $L_{0}$ represents a closed curve around the origin. For a homogeneous medium the 
isochrones $L_{0}$ are quartic ellipses confocal with the source as shown in Figure 3. The isochrones have a remarkable geometrical property illustrated in Figure 3 . Let $n$ be a unit vector at $A$ normal to $L_{0}$ on the plane of the source, and $n^{\prime}$ another unit vector normal to the tangent $T_{A}$ to $L_{0}$ and contained on a plane through this tangent and the observer. The angles $i_{1}$ and $i_{2}$, defined by $\cos i_{1}=(n, A 0)$ and $\cos i_{2}=\left(n^{\prime}\right.$, AP), satisfy the following Descartes-Snell law

$$
\frac{\sin i_{1}}{v}=\frac{\sin i_{2}}{c}
$$

The ray $A P$ may be interpreted as a seismic ray generated by the diffraction of the rupture ray at the isochrone line $L_{0}$. In order to prove the relation (11), we may consider as in Figure 3 a point $A^{\prime}$ located near $A$ on the tangent $T_{A}$. Point $A^{\prime}$ is outside the closed isochrone $L_{0}(P, t)$ and is located on another isochrone $L_{0}\left(P, t^{\prime}\right)$ with $t^{\prime}>t$. Thus, as we move $A^{\prime}$ away from $A$ along the tangent $T_{A}$ the travel-time $t^{\prime}$ increases. It is a minimum when $A^{\prime}$ coincides with $A$ and $t^{\prime}=t$. The equivalence

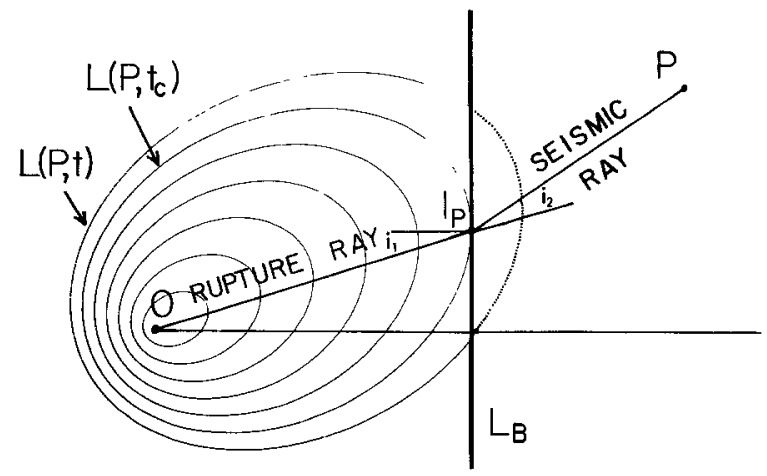

FIG. 4. Rupture ray and isochrone line. At time $t_{c}$, the isochrone $L(P, t)$ becomes tangent to the barrier $L_{B}$ at point $I_{P}$. At this same point $I_{P}$, the rupture ray is diffracted in the direction of $P$. For times greater than $t_{c}$, the missing segment of the isochrone is indicated by the dotted line.

of Fermat's principle for the minimum time and the Snell-Descartes law for diffraction proves the relation (11).

Now that the isochrone has been calculated, we can use the sifting property of the delta function to reduce the integral (7) over the fault surface into a line integral along the isochrone line $L$

$$
\boldsymbol{u}^{c}(P, t)=\frac{\mu D_{0}}{4 \pi \rho c^{3}} \int_{L} \boldsymbol{R}^{c} \frac{v}{D(1-v / c \cos \phi)} d l
$$

where $\phi$ is the angle between the radius vector of $A$ and the direction of the observer (see Figure 2). $L$ in (12) designates that part of the closed isochrone $L_{0}$ that is inside the barrier $L_{B}$, i.e., it contains the set of points $r_{1}(t, \theta)$ such that

$$
r_{1}(t, \theta)<r_{0}(\theta)
$$

Equation (12) provides a method to calculate near-field seismograms and accelerograms. Only two approximations have been made to generate (12), the first one is 
that the far-field Green function (1) be applicable, i.e., that the shortest wavelength $\lambda$ of interest be smaller than the minimum value of $D$. The other approximation, which may be relaxed by convolution, is that $\Delta \dot{u}$ be strongly concentrated at the rupture front (3). We will call asymptotic seismograms those calculated using approximation (12). Let us remark that since we are summing the radiation from a continuous distribution of sources on the fault, (12) is not strictly a high-frequency approximation. The proper high-frequency approximation to (12) will be obtained from the analysis of its discontinuities. This is the reason we prefer the name asymptotic approximation for (12) instead of high-frequency approximation.

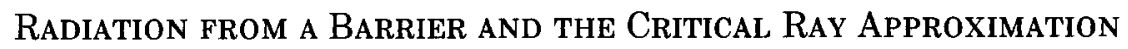

There are two major kinds of discontinuities in (12), the first ones are the starting phases radiated from the origin of rupture at 0 . These are weak singularities because the isochrone line $L$ shrinks to zero as the time $t \rightarrow R / c$, the arrival time for the radiation from the origin. Initial phases for the dislocation model $(2,3)$ behave like $t \cdot H(t)$ in displacement. Since these phases may be easily calculated by standard methods (see chapter 14 in Aki and Richards, 1980), we will concentrate on the strongest phases which are produced by the interaction of the rupture front and the barrier.

Let us examine the straight barrier first, as time increases from the starting phase arrival time, the isochrone $L_{0}(P, t)$ is closed and increases continuously in size. The displacement field calculated from (12) is, therefore, continuous. At a certain time $t_{\mathrm{c}}$, which we call the "critical" time, $L_{0}$ becomes tangent with the barrier $L_{B}$ at the point $I_{p}$ as shown on Figure 4. For times $t>t_{c}, L_{0}(P, t)$ cuts through the barrier and $L(P, t)$, which is the segment of $L_{0}(P, t)$ that is inside the barrier $L_{B}$, becomes an open line. This sudden change in the integration contour $L$ of (12) is the origin of the high-frequency waves diffracted by the barrier and which we call critical phases. If the rupture process were to stop completely after the interaction of the rupture front with the barrier, we would call these waves the stopping phases. The method we propose can deal with much more general situations than the stopping phases; this is why we prefer the same critical phase. For instance, we may study asperities where it is the $\operatorname{slip} D_{0}$, not the rupture velocity, that changes on the line $L_{B}$; or we may study very rapid changes in rupture velocity. The ray from $I_{P}$ to $P$ will be called a critical ray. If we can develop a method to trace these critical rays and to calculate the behavior of displacement (or acceleration) in their vicinity we would have a much simpler way to calculate high-frequency seismograms than equation (12).

Let us examine the kinematical properties of a critical ray and its associated wave front. At $I_{P}$, the isochrone is tangent to the barrier $L_{B}$ so that the normals to $L_{0}$ and $L_{B}$ coincide. Therefore, at $I_{P}$ the Snell-Descartes law for diffraction (11) may be reinterpreted in terms of the angles $i_{1}$ and $i_{2}$ that the rupture ray $O I_{P}$ and the critical ray $I_{P} P$ make with the local normals $n$ and $n^{\prime}$ to the barrier at $I_{P}$. Thus, $t_{c}$, the arrival time of critical phase from the barrier $L_{B}$ at $P$, is the minimum time for a ray that leaves the source with the rupture velocity $v$, is diffracted by the barrier, and then propagates to $P$ with the seismic velocity $c$. The diffracted ray that satisfies the Fermat's minimum time principle is the one that satisfies the diffraction law (11). The tracing and amplitude calculation of diffracted rays is a standard procedure of the geometrical theory of diffraction (Keller, 1962).

Now that we have constructed a critical ray that passes through $P$ we may 
determine the critical wave front generated by the barrier $L_{B}$. We proceed in the following way: for a fixed time $t_{c}$ we determine the locus of all the rays diffracted by the barrier which, for a given value of $\theta$, may be obtained from (10)

$$
D\left(t_{c}, \theta\right)=c t_{c}+c / v r_{0}(\theta) .
$$

Now for every value of $\theta$, we calculate the point on the barrier where the rupture ray intersects it. From this point we draw a cone of rays of length $D\left(t_{c}, \theta\right)$ and summit angle

$$
i_{2}=\arcsin \left(c / v \sin i_{1}\right) \text {. }
$$

Repeating this operation for all the angles $\theta$ we obtain the diffracted front at time $t_{c}$. Actually, because $c>v$, there is in general a maximum angle $\theta_{c}$ beyond which $i_{2}$ becomes complex. The critical wave front constructed in this manner has a cigar shaped form which is axially symmetric about the barrier line $L_{B}$. Figure 5 shows an example of a critical wavefront.

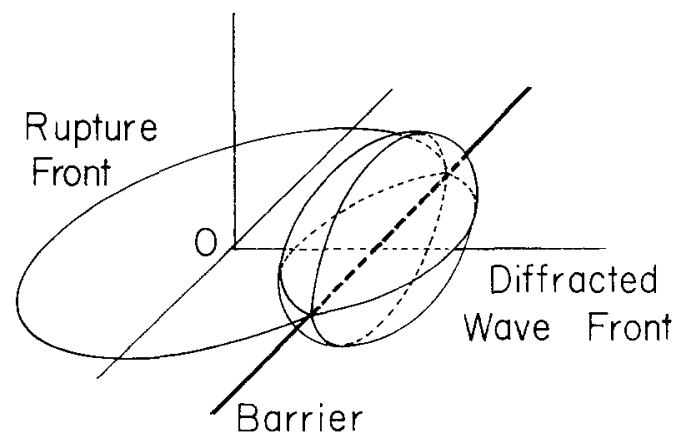

FIG. 5. Wave front radiated by the barrier. The circular rupture front with velocity $v$ is diffracted by the barrier, generating an axisymmetric wave front propagating with velocity $c$.

Now that we have traced the critical rays to the observer at $P$, we may determine the nature of the wave front discontinuities associated with the critical rays. The detailed derivation will be presented in the following section, here we give a simple qualitative explanation. When $t$ is slightly greater than $t_{c}$, the dicontinuity of the integral in (12) is due to the disappearance of the dashed line segment $\Delta L=L_{0}-$ $L$ in Figure 4 from the integration contour. The size of this segment is of order $\Delta L$ $\approx h \sqrt{t-t_{c}}$ where $h$ is a constant to be determined later. Since all the terms inside the integral are regular at $t_{c}$, we can approximate its singularity by

$$
\boldsymbol{u}(P, t)=\boldsymbol{F}\left(D, \phi, \boldsymbol{R}^{\mathrm{c}}\right) h \sqrt{t-t_{\mathrm{c}}} H\left(t-t_{\mathrm{c}}\right)
$$

where $\boldsymbol{F}$ is the value of the integrand in (12). For particle velocities, the singularity has the form

$$
d u(P, t) / d t=F h \frac{H\left(t-t_{c}\right)}{2 \sqrt{t-t_{c}}}
$$


and for acceleration

$$
d^{2} u(P, t) / d t^{2}=-F h \frac{H\left(t-t_{c}\right)}{4\left(t-t_{c}\right)^{3 / 2}}
$$

If the barrier has a general shape, described by $L_{B}$, the isochrone line may become tangent to it several times, generating at each tangent point a wave front discontinuity. Let us remember that the isochrone lines depend on the observation point and time, so that every observer will see at high frequencies a different set of critical points.

\section{The Dislocation Source Model with General Barrier Shape}

Let us consider the source model defined by the slip velocity function (3) buried in a homogeneous elastic medium. The barrier $r=r_{0}(\theta)$ may be a closed line of general shape surrounding the focus. Referring to Figure 2, the observer is at $P$, whose projection on the fault plane is $Q$. The focus $O$ is the origin of the cylindrical coordinates, and the polar angle in the fault plane is counted from the axis $O Q$. Point $A(r, \theta)$ is on the fault plane. We define: $h=P Q, d=O Q, r=O A, R=O P, \theta$ $=(O Q, O A)$ and $\phi=(O A, A P)$. Then $D(r, \theta)$, defined by (9), is the distance from $A(r, \theta)$ to $P$. We may now rewrite (7) in its explicit form

$$
\boldsymbol{u}^{c}(P, t)=\frac{\mu D_{0}}{4 \pi \rho c^{3}} \int_{0}^{2 \pi} d \theta \int_{0}^{r_{0}(\theta)} \boldsymbol{R}^{c} \frac{1}{D} \delta(t-\tau) r d r
$$

where $\tau$ is defined by (8). The isochrone lines $L_{0}(P, t)$ defined by (10) are closed lines surrounding $O$. For a given $t$ and a given $\theta$, there exists one and only one $r$, denoted $r_{1}(t, \theta)$, which verifies equation (10), because a radial line may intersect an isochrone line at only one point. Now let us change the variables $(r, \theta)$ to the new ones $(\tau, \theta)$. Then,

$$
\boldsymbol{u}^{c}(P, t)=\frac{\mu D_{0}}{4 \pi \rho c^{3}} \int_{0}^{2 \pi} d \theta \int_{R / c}^{\infty} \boldsymbol{R}^{c} \frac{1}{D} \delta(t-\tau) r_{1}(\tau, \theta) \partial_{\tau} r d \tau
$$

where the partial derivation $\partial_{\tau} r=\partial r / \partial \tau$ may be calculated from (8)

$$
\partial_{\tau} r=1 /\left(1 / v+\partial_{r} D / c\right)
$$

and $\partial_{r} D=-\cos \phi$ as may be seen from Figure 2. Doing the integral over $\tau$ we finally get

$$
\boldsymbol{u}^{c}(P, t)=\frac{\mu D_{0}}{4 \pi \rho c^{3}} f_{0}^{2 \pi} d \theta \boldsymbol{R}^{c} \frac{1}{D} \frac{v r_{1}(t, \theta)}{1-v / c \cos \phi}
$$

where the bar across the integral means that the integeral is taken only over the segments of the isochrone inside the barrier $L_{B}$. The factor $(1-v / c \cos \phi)^{-1}$ is the well-known directivity effect of the propagation of the rupture front. Note that (14) is the precise formulation of (12) in "Radiation from a Barrier and the Critical Ray 
Approximation", with $d l=r_{1} d \theta$. This integral is in general impossible to compute analytically, because we have to determine numerically the isochrone lines $r_{1}(t, \theta)$. It is, however, very simple to calculate numerically once the isochrone lines have been determined by ray tracing techniques. Seismograms calculated in this way will be called asymptotic and will be compared with calculations made by Bouchon (1982) with a discrete wave method.

Let us study now the origin of the wave front singularities of the integral over $\theta$ in (17). By definition of the barrier, $r_{1}(t, \theta)$ cannot be greater than $r_{0}(\theta)$ for a radiating point. This restriction appears in the integral (16) in the form of interruptions in the integration over $\theta$, which in turn create the wave front discontinuities that dominate the high-frequency behavior of the radiation. Let us consider the geometry of the barrier and isochrones shown in Figure 6. Initially, the isochrones $L_{0}(P, t)$ are continuous curves closed around the origin. As time increases, the isochrone eventually becomes tangent to the barrier at the point $I_{P}\left[r_{0}\left(\theta_{c}\right), \theta_{c}\right]$ at the critical time $t_{c}$. In the vicinity of $I_{P}, L_{0}$ cuts $L_{B}$ at two points defined by their polar angles $\theta_{1}$ and $\theta_{2}$. Two geometrical cases have to be considered, depending on the

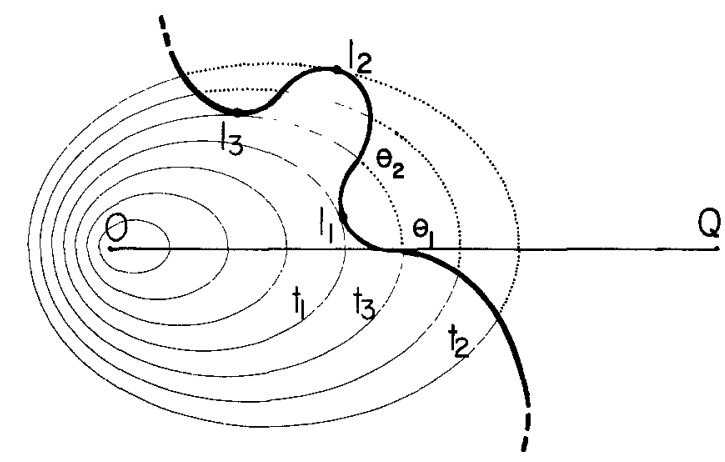

FIG. 6. Geometrical features for a barrier of general shape. The isochrone is tangent to the barrier line at $I_{1}, I_{2}$, and $I_{3}$, at times $t_{1}, t_{2}$, and $t_{3}$, respectively. The isochrone presents some missing segments whose number increases at $I_{1}$ and $I_{3}$, and decreases at $I_{2}$, depending on the relative radius of curvature. These singularities are related to the high-frequency radiation of the source.

relative curvature at $I_{P}$ of $L_{0}$ and $L_{B}$; they are illustrated in Figure 6 by the critical points $I_{1}$ and $I_{2}$. Just before the isochrone $L$ reaches the point $I_{1}$, the integral (16) is continuous. When time becomes greater than $t_{c}$, the isochrone in the vicinity of $I_{1}$ breaks into two segments. In the vicinity of $I_{2}$, on the other hand, a segment of $L$ disappears when $t$ increases beyond $t_{c}$. In either case for times $t \simeq t_{c}$, the discontinuity of the displacement field $\boldsymbol{u}$ in (16) may be aproximated by

$$
\boldsymbol{u}^{c}(P, t)=\mp \frac{\mu D_{0}}{4 \pi \rho c^{3}} \int_{\theta_{1}}^{\theta_{2}} d \theta \boldsymbol{R}^{c} \frac{1}{D} \frac{v r_{1}(t, \theta)}{1-v / c \cos \phi} .
$$

The upper sign applies to a critical point like $I_{1}$ of Figure 6 since in this case the singularity is due to the disappearance of the segment $\theta_{1}-\theta_{2}$ from the integral. The opposite sign applies to the case $I_{2}$ where the contour shrinks to zero as $t$ approaches $t_{c}$.

For $t \simeq t_{c}$, the segment of integration $\theta_{1}$ is small and the integral (17) may be 
approximated by

$$
\boldsymbol{u}^{c}(P, t)=\mp \frac{\mu D_{0}}{4 \pi \rho c^{3}} \boldsymbol{R}^{c} \frac{1}{D} \frac{v r_{1}\left(t_{c}, \theta_{c}\right)}{1-v / c \cos \phi_{c}} \quad\left(\theta-\theta_{2}\right)
$$

where $\boldsymbol{R}^{c}, D, \phi_{c}$, etc., are calculated at the critical point on the barrier. For the critical point $I_{1},(18)$ applies for $t>t_{c}$, while the opposite is valued for the critical point $I_{2}$. The discontinuities radiated by $I_{1}$ and $I_{2}$ are the Hilbert transforms of each other or, in other terms, the critical phase from $I_{1}$ is a minimum time phase while that from $I_{2}$ is maximum time (see Bernard and Madariaga, 1983).

In order to evaluate (18), we calculate $\left(\theta_{1}-\theta_{2}\right)$ in the vicinity of the critical angle $\theta_{c}$. Let us introduce the travel-time $T(\theta)$ of a rupture ray diffracted by the barrier $L_{B}$ in the direction of the observer. The diffraction angles $i_{1}$ and $i_{2}$ (see Figure 4) are not assumed to satisfy the relation (11). $T$ is given by

$$
T(\theta)=r_{0}(\theta) / v+D\left(r_{0}(\theta), \theta\right) / c
$$

where $D$ is defined by (9) and $r_{0}(\theta)$ is the equation of the barrier. For a given observer, $T$ is an extremum for $\theta=\theta_{c}$, the angle(s) that satisfies

$$
d T\left(\theta_{c}\right) / d \theta=0
$$

and the critical ray travel-time $t_{c}=T\left(\theta_{c}\right)$. It may be proven by a rather lengthy but straightforward procedure that the extremum condition for the travel-time $\theta_{c}$ may be recast in the more familiar form in terms of incidence and diffraction angles (11).

We may now calculate $\theta_{1}$ and $\theta_{2}$ in the vicinity of $\theta_{c}$ by means of the Taylor series for $T(\theta)$ in the vicinity of $\theta_{c}$

$$
T(\theta) \simeq t_{c}+\frac{1}{2} \frac{d^{2} T\left(\theta_{c}\right)}{d \theta^{2}}\left(\theta-\theta_{c}\right)^{2} .
$$

Since by definition the points $\theta_{1}$ and $\theta_{2}$ are located on the same isochrone of travel-time $t, T\left(\theta_{1}\right)=T\left(\theta_{2}\right)=t$ and we get for either $\theta_{1}$ or $\theta_{2}$

$$
\theta-\theta_{c}=\mp \sqrt{\frac{2\left(t-t_{c}\right)}{T^{\prime \prime}\left(\theta_{c}\right)}}
$$

the upper sign applies for $\theta_{1}$, the lower one for $\theta_{2}$ and $T^{\prime \prime}=d^{2} T / d \theta^{2}$. For $T^{\prime \prime}>0$, (22) gives $\theta_{1}$ and $\theta_{2}$ for $t>t_{c}$, this is the situation that prevails in the vicinity of $I_{1}$. For $T^{\prime \prime}<0, t$ has to be less than $t_{c}$ as for the critical phase radiated from $I_{2}$. We may now insert (22) into (18) and obtain the singularity associated with a critical phase. For a point like $I_{1}$, a minimum time phase, $T^{\prime \prime}\left(\theta_{c}\right)>0$ and we get

$$
\boldsymbol{u}^{c}(P, t)=-\frac{\mu D_{0}}{4 \pi \rho c^{3}} \boldsymbol{R}^{c} \frac{2 \sqrt{2} v}{1-v / c \cos \phi} \frac{r_{1}}{D} \frac{\sqrt{t-t_{c}}}{\sqrt{\left|T^{\prime \prime}\right|}} H\left(t-t_{c}\right)
$$

where $T^{\prime \prime}, \boldsymbol{R}_{c}, r_{1}, D$, and $\phi$ are calculated at the critical point $I_{1}$. For a maximum 
time critical phase, like $I_{2}, T^{\prime \prime}<0$ and

$$
\boldsymbol{u}^{c}(P, t)=+\frac{\mu D_{0}}{4 \pi \rho c^{3}} \boldsymbol{R}^{c} \frac{2 \sqrt{2} v}{1-v / c \cos \phi} \frac{r_{1}}{D} \frac{\sqrt{t_{c}-t}}{\sqrt{\left|T^{\prime \prime}\right|}} H\left(t_{c}-t\right)
$$

where $T^{\prime \prime}, \boldsymbol{R}_{c}, r_{1}, D$, and $\phi$ are calculated at the critical point $I_{2}$. As we have already suggested, the time dependence of (24) is the Hilbert transform of that of (23). The second derivative of the travel time is the usual geometrical spreading for twodimensional waves. It may be recast in a form that is closer to that used in geometrical diffraction theory but the algebra is rather long and $(23,24)$ is more suitable for numerical calculation. For a uniform elastic medium, $T^{\prime \prime}$ may be directly calculated by differentiation of the travel time (19).

Equations (23) and (24) give the high-frequency part of the displacement $u$ : the critical time $t=t_{c}$ corresponds to the arrival time of the ray reaching $P$ and passing through $I_{P}$. So the critical point $I_{P}$ seems to radiate the high-frequency signal. Remember that the position of the critical points on a barrier depends on the observer position $P$.

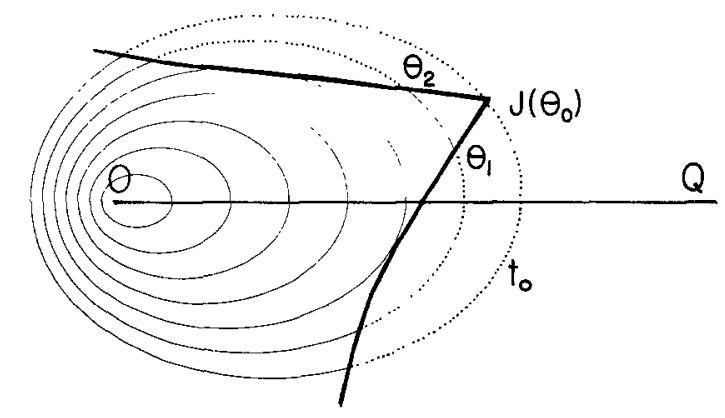

FIG. 7. Broken barrier line. The barrier presents an angular point $J\left(\theta_{0}\right)$ at time $t_{0}$, which is responsible for high-frequency radiation similar to the starting phase at the focus.

\section{Extension and Limits of the High-Frequency Method}

The results of "The Dislocation Source Model with General Barrier Shape" may be adapted to deal with more realistic seismic sources than a dislocation expanding with constant rupture velocity and stopping abruptly on a regular barrier line. In this section, we discuss the following two generalizations.

1. The barrier line presents some angular points.

2. The slip velocity is that of a crack model and has the characteristic inverse square root singularity (Madariaga, 1977) near the rupture front.

Let us consider first the case of a broken barrier line. Physically, that means that on some points of the barrier, the curvature radius is shorter than the wavelength. We call $J\left[r_{0}\left(\theta_{0}\right), \theta_{0}\right]$ an angular point of the barrier (see Figure 7 ). The rupture front reaches point $J$ at time $t_{0}$. The angles $\theta_{1}$ and $\theta_{2}$ are equal when $t=t_{0}$, but the isochrone line is not tangent to the barrier, as it was in the case of a critical point, and the travel time $T\left(\theta_{0}\right)$ is not an extremum. The Taylor expansion (21) becomes, to first order

$$
T(\theta)=t_{0}+T^{\prime}\left(\theta_{0}\right)\left(\theta-\theta_{0}\right)
$$


so that, since $\theta_{1}$ and $\theta_{2}$ are on the same isochrone of travel-time $t$,

$$
\theta_{1}-\theta_{2}=2 \frac{t-t_{0}}{T^{\prime}}
$$

Inserting this relation into the standard expression (18), we get

$$
\boldsymbol{u}^{c}(P, t)=+\frac{\mu D_{0}}{4 \pi \rho c^{3}} \boldsymbol{R}^{c} \frac{2 v}{1-v / c \cos \phi} \frac{r_{1}}{D} \frac{t_{0}-t}{T^{\prime}} H\left(t_{0}-t\right)
$$

where $T^{\prime}, \boldsymbol{R}^{c}, r_{1}, D$, and $\phi$ are calculated at the angular point $I_{0}$. Thus, the presence of a corner in the barrier line produces a discontinuity in the displacement $\boldsymbol{u}$ of the form

$$
\boldsymbol{u}^{c}(P, t) \alpha H\left(t_{0}-t\right)\left(t_{0}-t\right) \quad \text { or } \quad H\left(t-t_{0}\right)\left(t-t_{0}\right)
$$

The spectral amplitude is of the order $1 / \sqrt{\omega}$ times the spectra of $\boldsymbol{u} \approx$ $H\left(t-t_{c}\right) \sqrt{t-t_{c}}$, therefore the contribution of the angularities of the barrier to the high-frequency radiation is weaker than the contribution of the critical points, and may be neglected to the first order.

Note that the starting phase for $t \simeq R / c$ gives a similar displacement $u(P, t) \alpha$ $H(t-R / c)(t-R / c)$. Another important consequence is that for any point $Q$ in space, the angular point $J$ is of course fixed so that, apart from the directivity term, the high-frequency radiation from a corner does not depend on the position of the observation point $Q$ as it was the case with critical phases where the critical point moves along the barrier as the observation point is displaced.

The second extension that we want to discuss is a quasi-dynamical model (Boatwright, 1982) for slip velocity since a crack model is more satisfactory in a physical sense than a dislocation model. As the rupture front propagates, the slip velocity may be expressed as

$$
\Delta u(r, \theta, t)=(t-r / v)^{-1 / 2} H(t-r / v)
$$

near the front. Let us consider two particular behaviors of the rupture front when it reaches a barrier.

In the first model, we take

$$
\Delta u(r, \theta, t)=\frac{H(t-r / v)}{\sqrt{t-r / v}} H\left(r_{0}(\theta)-r\right)
$$

meaning that slip continues indefinitely once the rupture stops at the barrier line. An alternative model is

$$
\Delta u(r, \theta, t)=\frac{H(t-r / v)}{\sqrt{t-r / v}} H\left(r_{0}(\theta)-v t\right)
$$

which means that the crack stops simultaneously on the whole radial line of angle $\theta$ when the rupture front reaches the barrier along that line. Between these two 
extreme models, unfortunately, it seems difficult to construct a realistic model with stopping phases without loosing the simplicity of the analytical expression of slip velocity.

For the first extreme model, the high-frequency displacement is the one obtained in (23) or (24), convolved with $(t-r / v)^{1 / 2}$. Then if $T^{\prime \prime}>0$,

$$
u(P, t) \approx\left(t-t_{c}\right) H\left(t-t_{c}\right)
$$

and

$$
d^{2} u(P, t) / d t^{2} \approx \delta\left(t-t_{c}\right)
$$

while for $T^{\prime \prime}<0$ we obtain the Hilbert transformed pulses. For example, the acceleration pulse behaves like

$$
d^{2} u(P, t) / d t^{2} \approx H\left(t-t_{c}\right) /\left(t-t_{c}\right) .
$$

The high-frequency acceleration radiated by a propagating crack stopping abruptly is well described by a superposition of $\delta$-like and $1 / t$-like impulses, generated at the critical points on the barrier.

These results are compatible with observational acceleration spectra, for which the high-frequency part is usually flat. If the jump of the rupture velocity is not instantaneous, but takes a time $\Delta t$, the spectral amplitude of acceleration should break down for frequencies greater than $f_{\max }=1 / \Delta t$.

The interest of the method presented here is that once we have solved for the radiation in a uniform medium, we may simply apply ray theory to propagate the high-frequency signal in a more realistic heterogeneous attenuating medium.

\section{COMPaRISON With Discrete WaVenumber ACCELERograms}

We shall now apply the results of the previous sections to the calculation of synthetic accelerograms for the Gilroy 6 recording of the 6 August 1979 Coyote lake earthquake in California. This event was studied by Bouchon (1982) who proposed a dislocation model for the source and calculated synthetic accelerograms with his discrete wavenumber method. His model is a vertical strike-slip fault as shown in Figure 8. Rupture starts at $9.5 \mathrm{~km}$ depth and propagates self-similarly with a constant rupture velocity of $2.6 \mathrm{~km} / \mathrm{sec}$. Slip is constant (dislocation model) and equal to $D_{0}=21 \mathrm{~cm}$. The final fault shape is defined by a rectangular barrier where rupture stops abruptly. The Gilroy 6 station was practically on the fault trace, 10 $\mathrm{km}$ away from the epicenter. The medium consists of an upper layer $1.75 \mathrm{~km}$ thick, with a shear velocity of $2.4 \mathrm{~km} / \mathrm{sec}$, and a density of $2.6 \mathrm{gm} / \mathrm{cm}^{3}$, overlying an elastic half-space with a shear velocity of $3.5 \mathrm{~km} / \mathrm{sec}$ and density $2.8 \mathrm{gm} / \mathrm{cm}^{3}$.

Let us first apply the theory of critical rays. We trace the critical rays using standard ray theory for a layer over a half-space and relation (11) for diffraction by the barrier. For each segment of the barrier, there is a single critical point that diffracts a particular ray in the direction of the station. We denote by $I_{1}, I_{2}, I_{3}$, and $I_{4}$ these four critical points on Figure 9. The rays through these four points will be used to generate high-frequency seismograms.

Next we calculated the isochrones for regular increments of the observation time. They were obtained by a numerical solution of equation (10) where $D / c$ was replaced 
by the appropriate expression for the travel time in a layer over a half-space. As seen in Figure 10, the isochrones, as expected, are tangent to the barriers at the four critical points. Since at any point on the fault plane the radial distance between neighboring isochrones is proportional to the directivity factor, the area that radiates the higher amplitudes is the elongated sector pointing from the source to the observation point. This explains why, as will be shown later, the critical phase $I_{2}$ dominates high-frequency radiation.

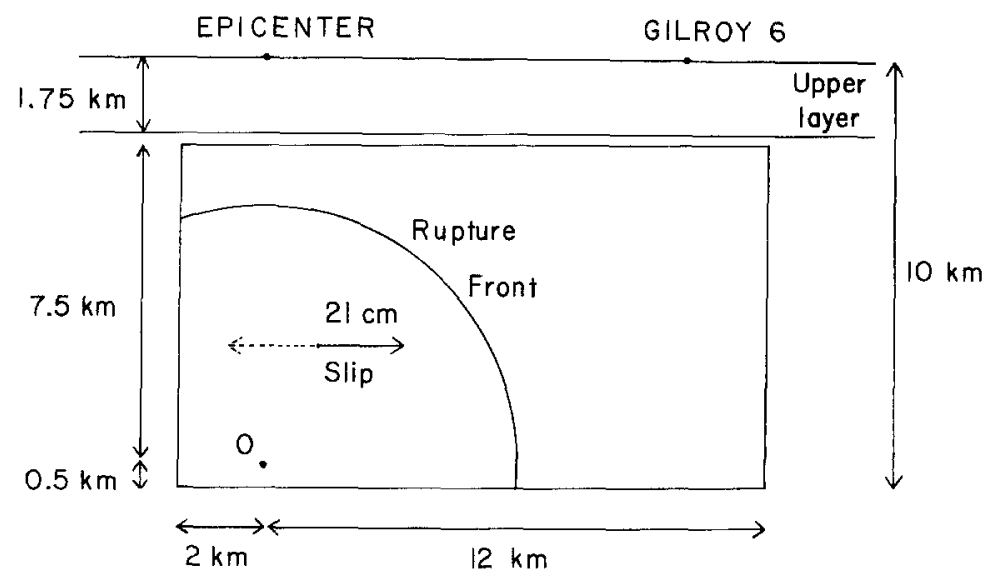

FIG. 8. Rectangular source model. The fault plane is vertical and the focus at depth $9.5 \mathrm{~km}$. The rupture front propagates with contant velocity $2.6 \mathrm{~km} / \mathrm{sec}$ and stops on the rectangular barrier $8 \mathrm{~km} \times$ $14 \mathrm{~km}$, whose top is at $2 \mathrm{~km}$ depth. The station (Gilroy 6) is at $10 \mathrm{~km}$ from the epicenter, on the fault plane. The slip inside the rupture front is $21 \mathrm{~cm}$ (dislocation) in the horizontal direction (strike-slip). The medium has an upper layer $1.75 \mathrm{~km}$ thick.

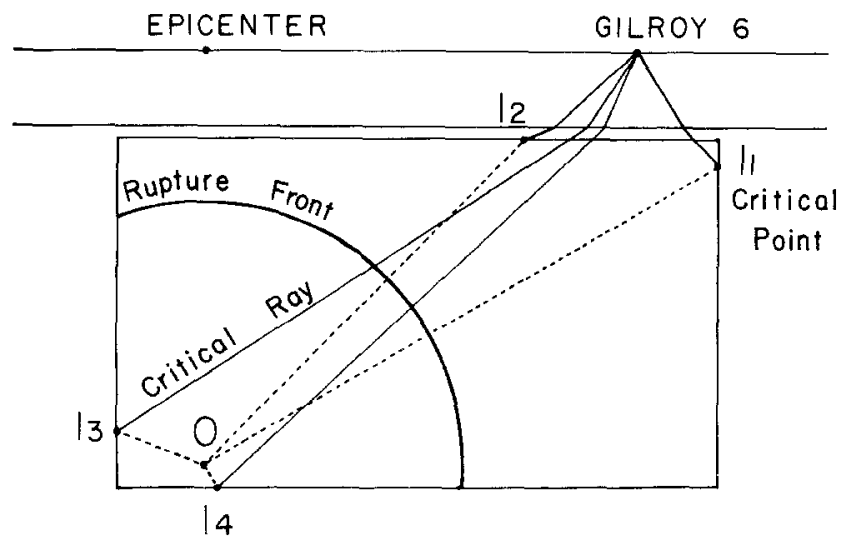

FIG. 9. Critical rays and critical points for the rectangular barrier model. Rupture rays are associated with the circular rupture front and are diffracted by the barrier in the direction of the station. The four minimum travel time rays, diffracted at the critical points $I_{1}, I_{2}, I_{3}$, and $L_{4}$, are the critical rays that dominate the high-frequency motion at Gilroy 6.

We may now compute synthetic displacement records by the two methods that we have proposed: integration along the isochrones and the critical ray approximation. Since station Gilroy 6 is practically on the fault plane, only $S H$ waves with displacement perpendicular to the fault have to be considered. We computed the displacement and velocity records shown on the top in Figure 11 using equation (16). We call these asymptotic seismograms since the only approximation involved 


EPICENTER GILROY 6

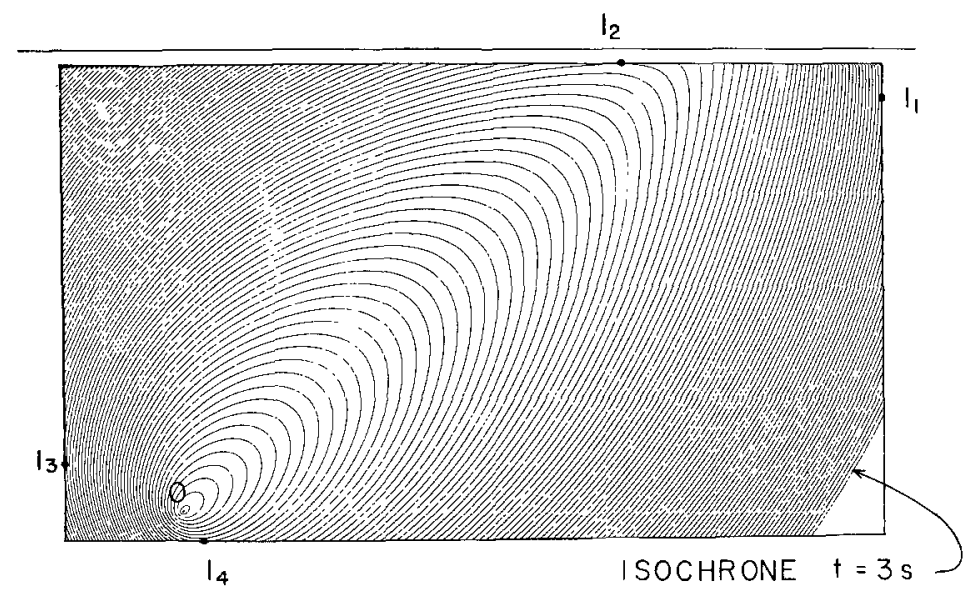

FIG. 10. Isochrone for the rectangular barrier model. The isochrones are plotted for a regular increment of the observation time. They are tangent to the barrier at points $I_{1}, I_{2}, I_{3}$, and $I_{4}$, which are also the critical points of Figure 9.

DISPLACEMENT
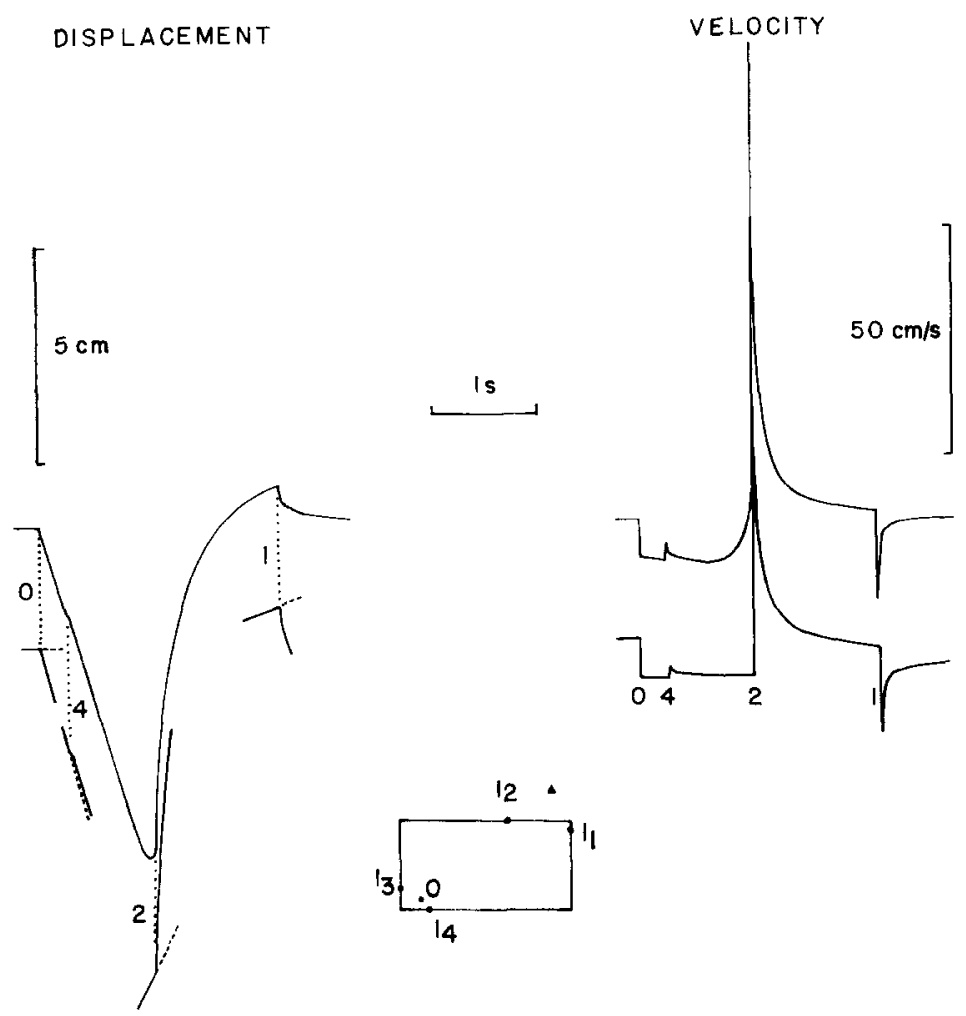

FIG. 11. Comparison between the asymptotic method and the critical ray method. For the displacement, the fit is good near the arrival times of the high-frequency phases $0,1,2$, and 4 radiated from $0, I_{1}, I_{2}$, and $I_{4}$, respectively. We did not plot phase 3 , radiated by $I_{3}$, because its arrival time is very close to that of phase 2, and its amplitude is even weaker than phase 1. For velocity, there are no significant differences, except just before phase 2 , where the rapid increase of $u$ and $d u / d t$ is explained by the proximity of the interface to point $I_{2}$. 
in their calculation is the use of the far-field asymptotic approximation to the Green function. Particle velocity was calculated by numerical differentiation of displacement. Theoretically, these asymptotic records are valid for frequencies greater than $1.5 \mathrm{~Hz}$ in order that all wavelengths be shorter than the distance to the fault. We clearly identify in the synthetics of Figure 11 the starting phase (0) and the highfrequency phases generated at the critical points $I_{1}$ and $I_{2}$. The radiation from $I_{3}$ and $I_{4}$ is less easily identified because it is very weak. Just before the arrival time of the phase radiated by $I_{2}$, the displacement increases rapidly. This is not a barrier effect, but is explained by the very short distance between the interface and the top of the barrier: the consequence is a very rapid decrease with time of the transmission

\section{DISPLACEMENT}

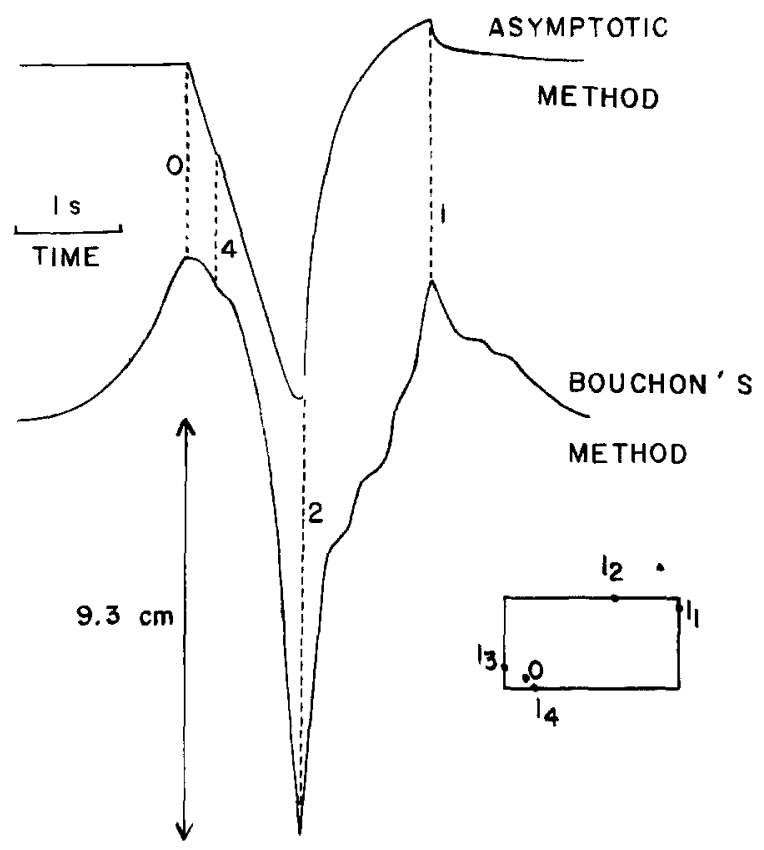

FIG. 12. Comparison between the asymptotic method and Bouchon's full numerical method. The asymptotic displacements calculated by the two methods show a similar overall shape. Phases $0,1,2$, and 4 in the asymptotics appear clearly in the complete solution, and their arrival times coincide perfectly. The arrival before phase 0 in Bouchon's synthetic is due to near-field low frequencies, which would be reduced any may be neglected in velocity and acceleration synthetics.

coefficient near $I_{2}$. It is also interesting to note that the radiation from the corners is very weak. This is in contrast to the Haskell model where radiation comes mainly from the corners as shown by Madariaga (1978).

In Figure 11, we also plot the high-frequency displacement and velocity calculated by the critical ray approximation [equation (23)]. This is the generalization of the stopping phase approximations that Bernard and Madariaga (1983) used to approximate the radiation from a circular fault model. We may compare on Figure 11 the results obtained by the asymptotic method and the critical ray approximation. For displacement, the fit is good only near the singularities; for velocity, on the other hand, the two functions match almost at all times. We conclude that, for velocity, 
the much simpler critical ray approximation is as good at high-frequency as the more expensive method of integration along the isochrones.

Let us now compare in Figure 12 the asymptotic displacement with the displacement computed numerically by Bouchon (1982) for the rectangular fault. $\mathrm{He}$ discretized the fault into elementary sources and calculated displacement superposing the full near-field radiation for each of these sources. In spite of the different theoretical limits of validity for the two methods, $f<3.2 \mathrm{~Hz}$ for Bouchon and $f>$ $1.5 \mathrm{~Hz}$ for the asymptotic method, the two solutions are very similar. The principal high-frequency phases are clearly identified, and the general form even at low frequencies is very similar. The asymptotic solutions appear to be valid down to frequencies of $1 \mathrm{~Hz}$ in this case. The principal difference between our results and Bouchon's is the rapid increase of $u$ just before phase 2 which does not appear in his synthetic. This difference is probably due to the discretization of the source in Bouchon's model. In fact, the top row of elementary sources in his discrete model is deeper than the nominal top of the fault so that they are farther from the interface, and the rapid change in reflection coefficient that causes the increase in $u$ in our synthetics is absent from his model.

Our results permit us to compare three methods to calculate synthetics in the vicinity of a fault. First, the most complex, numerical integration of complete nearfield Green functions (Bouchon, 1982). Second, numerical integration along isochrones derived from the use of the far-field approximation for the Green functions and, third, the critical ray approximation. It appears that for displacement, the first two methods give practically the same result. For velocity, the critical ray approximation works almost as well as the asymptotic method. For acceleration calculations, the critical ray approximation is definitely superior allowing - at least in principle-the frequency resolution to increase without limit. In practice, attenuation and source properties will limit the resolution.

The rectangular source model discussed here gives a synthetic displacement which fits well the general shape of the Gilroy 6 record for the Coyote Lake earthquake. Bouchon had then to modify the main parameters of his model to improve the fit to the data. The use of the methods proposed here could be of great help in understanding how the different source parameters control the synthetic record. It would then be possible to develop inversion procedures to obtain the source parameters.

\section{CONCLUSIONS}

A theoretical study of the radiation from a moving dislocation loop stopping at a barrier of general shape has revealed a number of important geometric and kinematic features which we describe with the following three concepts: (1) isochrone lines, the locus on the fault of those points whose radiation reaches the observer at a given time. This is a very general concept valid in general heterogeneous media which depends only on the use of the ray theoretical approximation to the Green function for every point on the fault. (2) A rupture ray is a ray that propagates initially on the fault plane with the local rupture velocity, is diffracted by a barrier, and then propagates to the observation point with the elastic wave velocity. (3) Critical points, which are the points on a barrier from which is diffracted the rupture ray with minimum travel time, we call this ray a critical ray. The critical points are also the points of tangency between an isochrone line and a barrier. The critical 
ray satisfies an extension of Snell's law for the diffraction of the rupture ray into a seismic ray by the barrier. Using ray tracing techniques, there critical rays (stopping phases if we are dealing with an unbreakable barrier) may be computed and used to estimate accelerograms. These concepts may be generalized for more complex rupture front propagation, including rupture velocity jumps and gradients. Based on these results, two methods of approximation for near-source records were proposed, in the first, an asymptotic approximation is obtained which gives the displacement and velocity as a single integral along an isochrone for every time point. The other method or critical ray approximation, which is appropriate for the synthesis of velocity and acceleration, gives closed-form expressions for the behavior near the wave fronts. These approximations are certainly more efficient for computer evaluation than the full numerical methods which are currently in use. Even more interesting is that it appears from our results that at high frequencies, the fault reduces to a finite number of critical points from which critical rays are emitted that completely dominate the strong motion. These critical points depend on the position of the observation point with respect to the fault barriers. This may explain the great complexity of accelerograms and their variability from one station to another. It does also suggest several ways in which the inversion of source parameters from near-field observations may be approached.

\section{ACKNOW LEDGMENTS}

We thank Dr. Michel Bouchon for many enlightening discussions about near-field modeling. Careful reading and healthy criticism by Dr. J. Boatwright has helped to produce what we hope is an improved presentation of our results. The final version of this paper was completed while $\mathrm{R}$. Madariaga was a Visiting Scientist at the Lamont-Doherty Geological Observatory of Columbia University. This work was supported by Institut National de Géophysique under ATP Sismogénèse and by the Bureau de Recherche Géologique et Minière (B.R.G.M).

\section{REFERENCES}

Achenbach, J. D. and J. A. Harris (1978). Ray methods for elastodynamic radiation from a slip zone of arbitrary shape, J. Geophys. Res. 83, 2283-2291.

Aki, K. and P. Richards (1980). Quantitative Seismology, W. H. Freeman, San Francisco, California.

Archuleta, R. J. and S. H. Hartzell (1981). Effects of fault finiteness on near-source ground motion, Bull. Seism. Soc. Am. 71, 939-957.

Bernard, P. and R. Madariaga (1983). High frequency seismic radiation from a buried circular crack, Geophys. J. R. Astr. Soc. (in press).

Boatwright, J. (1982). A dynamic model for the far field acceleration, Bull. Seism. Soc. Am. 72, 10491068.

Bouchon, M. (1979). Discrete wave number representation of elastic-waves in three-space dimensions, J. Geophys. Res. 84, 3609-3614.

Bouchon, M. (1982). The rupture mechanism of the Coyote lake earthquake of August 6, 1979 inferred from near field data, Bull. Seism. Soc. A. 72, 745-759.

Bouchon, M. and K. Aki (1976). Discrete wave number representation of elastic wave fields, Bull. Seism. Soc. Am. 67, 259-277.

Campillo, M. (1983). Numerical evaluation of the near-field, high-frequency radiation from quasidynamic circular faults, Bull. Seism. Soc. Am. 73, 723-734.

Hanks, T. C. (1982). fmax, Bull. Seism. Soc. Am. 72, 1867-1879.

Keller, J. B. (1962). Geometrical theory of diffraction, J. Opt. Soc. Am. 52, 116-130.

Madariaga, R. (1977). High frequency radiation from crack (stress drop) models of earthquake faulting, Geophys. J. R. Astr. Soc. 51, 625-651.

Madariaga, R. (1978). The dynamic field of Haskell's rectangular dislocation fault model, Bull. Seism. Soc. Am. 68, 869-887. 
Papageorgiou, A. J. and K. Aki. (1982). A specific barrier model for the quantitative description of inhomogeneous faulting and the prediction of strong ground motion. II. Applications of the model, Bull. Seism. Soc. Am. 73, 953-978.

L.E.G.S.P., AssociÉ AU C.N.R.S. 195 Institute de Physique du Globe Université Pierre ET Marie Curie 4, Place Jussieu 75230 PaRis CedeX 05 France

CONTRIBUTION No. 713

Manuscript received 20 June 1983 